\title{
Renin-Angiotensin Inhibition in Combating Malignancy: A Review
}

\author{
TALMA ROSENTHAL ${ }^{1}$ and IRENE GAVRAS ${ }^{2}$ \\ ${ }^{1}$ Department of Physiology and Pharmacology, Hypertension Research Unit, \\ Sackler School of Medicine, Tel-Aviv University, Tel-Aviv, Israel; \\ ${ }^{2}$ Hypertension and Atherosclerosis Section, Boston University, School of Medicine, Boston, MA, U.S.A.
}

\begin{abstract}
Our previous review of the literature assessed the existing knowledge (until 2000) about the possible link between angiotensin-converting enzyme inhibitors (ACEIs) and factors influencing the development of malignancies. We reviewed the literature for reports of statistical associations (or lack thereof) between ACEi treatment and incidence of specific cancers (e.g. breast, gastrointestinal, and skin). We concluded then that results from the epidemiological studies are conflicting, even taking the different methodology and endpoints into consideration, and thus inconclusive. Further investigation is needed beyond the observation period of most of these studies, and additional experimental studies are needed also to study the mechanisms by which agents blocking the renin-angiotensin system may obtain their inhibitory effect on tumor growth and metastasis. The present review elaborates further with more recent evidence from numerous human clinical studies from the past two decades (including large epidemiological studies, and long-term prospective and retrospective studies) on a protective association between ACEi treatment and the prognosis of patients with specific cancer types, malignancy characteristics or stage. Moreover, treatment with ACEI/angiotensin receptor blockers represents an adjuvant therapy with synergistic effects to chemotherapy and may improve patient outcomes (i.e. progression-free survival, and prolonged overall survival) in different types of cancers.
\end{abstract}

This article is freely accessible online.

Correspondence to: Professor Talma Rosenthal, Department of Physiology and Pharmacology, Hypertension Research Unit, Sackler School of Medicine, Tel-Aviv University, Tel-Aviv, Israel. E-mail: rtalma@tauex.tau.ac.il

Key Words: ACE inhibitors, malignancy, renin-angiotensin system, review.
The renin-angiotensin system (RAS) is a hormonal system in which active peptide angiotensin II (Ang II) signaling increases cell proliferation and stimulates neovascularization. A growing body of evidence suggests that angiotensin II signaling promotes vascular endothelial growth factor (VEGF)-mediated angiogenesis in malignancy by directly affecting tumor and stromal cells and by indirectly modulating the growth of vascular cells during angiogenesis (1).

Angiotensin-converting enzyme inhibitors (ACEIs) and angiotensin receptor blockers (ARBs) are two widely used types of hypertension medications. Since the main mechanism of ACEIs and ARBs is to act as antagonists of the RAS, they inhibit its effect on angiogenesis and thus diminish the growth of induced cancer and may reduce the risk of cancer over time. However, there are additional potential mechanisms mediated via the internalization and endonuclear localization of the ACE that can be affected by these drugs in various cell types, which may be relevant to certain aspects of carcinogenesis (2). In both patients and animal models ACEIs reportedly not only suppressed tumor growth, but also attenuated the process of carcinogenesis in which angiogenesis is involved (3).

The first such hypothesis-generating findings presented by Lever et al. in retrospective analysis of 5,207 hypertensive patients showed long-term treatment ( $>3$ years) with ACEI resulted in reduced relative risk of incidence of breast and lung cancer (4).

Our previous review of the literature assessed the existing knowledge (until 2000) about the possible link between ACEIs and factors influencing the development of malignancies (5). The present review elaborates further with more recent evidence from numerous human clinical studies from the past two decades (including large epidemiological studies, and longterm prospective and retrospective studies) on a protective association between ACEi treatment the prognosis of patients with specific cancer types, malignancy characteristics or stage. Moreover, treatment with ACEI/ARB represents an adjuvant 
therapy with synergistic effects to chemotherapy and may improve patient outcomes (i.e. progression-free survival, and prolonged overall survival) in different types of cancer.

\section{Blockade of RAS in Experimental Animal Models}

The effects of angiotensin II blockade with either ACEI or $\mathrm{ARB}$ alone, and in combination were investigated in a murine model of renal cell carcinoma (RCC) (6). Notably, human patients are never treated with a combination of $\mathrm{ACE}$ and ARB together. The results of this animal experiment showed treatment significantly reduced tumor growth and the number of lung metastases in treated mice when compared with controls (no treatment). In contrast, treatment with ARB or ACEI alone resulted in significantly smaller tumors as compared to those treated with double blockade, thus suggesting that in this experimental animal model, blockade of RAS reduces RCC proliferation and metastatic capacity (6). Similar results were found in another experimental animal model of RCC. In uni-nephrectomized rats, the histopathology of carcinogenesis in remnant kidneys was manifested by atypical proliferation and carcinoma in situ; compared to the controls, in the treatment group of uninephrectomized rats, ACEI, and ARB significantly prevented renal carcinogenesis (7).

\section{Clinical Observations}

The effect of RAS inhibitors (RASi) on clinical outcomes of patients with different types of cancer has been extensively studied in recent years. We aimed to review the literature reporting statistical associations (or lack thereof) between treatment with ACEI or ARB and incidence of specific cancer types.

Conflicting results have been shown in large epidemiological studies assessing whether ACEIs and ARBs have potentially protective effects against cancer risk. Two large population-based studies, from Denmark (10) and a recent study from 2017 in the UK (11), found no such association. Conversely, two large epidemiological studies published in 2014, conducted in the UK (12) and in Taiwan (13), demonstrated an association between ACEI/ARB use and a reduced risk of developing cancer. In a large population-based cohort of 17,897 patients from Denmark, treatment with ACEI was not associated with a reduced risk of developing cancer (10). Similar results were shown by Mandilaras et al. in a UK population-based cohort of 547,566 patients in 2017 (11). Compared to use of other hypertensive drugs, a cumulative duration of 1-3 years of ARB use was associated with a $38 \%$ (odds ratio $=0.62,95 \%$ confidence intervaI $(\mathrm{CI})=0.41-0.94)$ reduced risk of pancreatic cancer, although use of ACEIs and ARBs did not reduce overall incidence of pancreatic cancer.
Conversely, two large epidemiological studies published in 2014, conducted in the UK (12) and in Taiwan (13), demonstrated a positive association between ACEI/ARB use and a reduced risk of developing cancer. A large UK cohort of 2,847 patients who developed CRC were matched to 28,239 controls. Compared to the use of other antihypertensive medications, long-term ( $>3$ years) cumulative use of high-dose ACEI or ARB was associated with a reduced incidence of CRC (12). A similar positive association was shown in a large population-based cohort of 297,688 individuals in Taiwan (13); use of ACEI or ARB was associated with a lower overall risk of cancer.

In accordance with findings in the two aforementioned large epidemiological studies on CRC, a systematic review with meta-analysis of six observational studies totaling 113,048 individuals and four case-control studies found a $6 \%(95 \% \mathrm{CI}=0.90-0.99)$ reduced risk of CRC in ACEI/ARB users (14).

A meta-analysis of 17 observational studies (case-control studies and cohort studies) with a total of 3,833,261 participants, of whom 289,858 were ACEI/ARB users with long follow-up (2.4-7.8 years), concluded monotherapy with ACEI/ARB might have protective effects on cancer, showing reduced incidence and mortality from cancer (15).

A prospective population-based study (based on data from the Rotterdam Study) included 7,983 individuals with a mean follow-up of 9.6 years, concluded that those with the highactivity DD genotype for $A C E$ had an increased risk of developing breast cancer, while long-term and high-dose ACEI treatment showed a protective effect against developing CRC, lung, breast, and prostate cancer in those with DD genotype (16). Another study investigated whether ACE gene polymorphisms are associated with increased susceptibility to oral cancer (17). They found that compared to a control group, patients with oral cancer had a higher frequency of the DD genotype. Moreover, individuals with the DD genotype had a 5.46-fold higher risk of developing oral cancer.

\section{Determinants of RASi Efficacy}

There are several plausible determinants of RASi efficacy as detailed in the studies below. Multiple studies found RASi use was associated with better patient outcomes, whereas in other studies, no association was found. This possibly suggests that responses to RASi treatment are dependent on tumor type and on certain tumor characteristics.

In accordance with findings from the aforementioned experimental animal models, a recent review found the types of cancer for which RASi therapy was associated with better patient outcomes included renal and hepatic cancer (18). Keizman et al. demonstrated such an association; in a multicenter pooled analysis among 278 patients with metastatic RCC, $106 \mathrm{ACEI} / \mathrm{ARB}$ users experienced longer progression- 
free survival (19). Another pooled analysis by McKay et al. demonstrated this positive association; among 4,736 patients with metastatic RCC, 1,487 ACEI/ARB users had longer overall and progression-free survival compared to users of other antihypertensive medications (20). Conversely a U.S. population-based case-control study of RCC among 2,304 participants found little evidence of an association with use of ACEIs on RCC risk (21).

A recent review of patients with hepatocellular cancer (HCC) treated with RASi showed improved outcomes in two randomized control studies, showing significantly reduced recurrence rates of carcinoma (18). Another randomized control study found combination of branched-chain amino acids and ACEI significantly suppressed the cumulative recurrence of HCC (22). Indeed, in the pathophysiology of liver cirrhosis the angiotensin II-angiotensin II receptor type 1 axis has a crucial role as RASi treatment may affect improvement of both liver fibrosis and portal hypertension. Conversely, a case-control study of 224 patients each matched with 10 controls found neither any use or long-term use of ACEI had a protective effect against the development of HCC (23).

RASi therapy was shown to have a clinical benefit in early-stage (e.g. resected urinary tract cancer) and slowly progressing cancer (e.g. prostate cancer). Positive clinical outcomes in patients after resection of bladder cancer were also demonstrated by Blute et al. among 340 patients; 143 ACEI/ARB users had longer recurrence free survival (24). Moreover, Yoshida et al. showed ACEI/ARB use in patients with bladder cancer was an independent prognostic factor for both longer cancer-specific and 5-year overall survival (25). Finally, in a study by Alashkham et al. among 558 patients with local/locally advanced prostate cancer, 103 ACEI/ARB users had a reduced risk of biochemical recurrence (26).

RASi therapy has also shown positive clinical outcomes in advanced stages (e.g. metastatic lung cancer) and highly aggressive tumor types (e.g. glioblastoma and pancreatic ductal adenocarcinoma (PDAC). Contrary to the aforementioned epidemiological study by Mandilaras et al. (11), a recent study by Liu et al. in patients with PDAC showed benefits of RASIs (27). Their study with 794 patients with PDAC demonstrated that in 297, chronic ACEI/ARB use was associated with longer overall survival (independent of neoadjuvant chemotherapy) in those with resected and locally advanced non-metastatic PDAC. Moreover, they performed an RNA-Seq gene expression profiling which suggested that the improved survival associated with RASi therapy is possibly due to inhibition of tumor progression and enhanced antitumor immunity in primary PDAC (27).

While the aforementioned cancer and tumor types may belong to those responsive to RASi treatment, past studies show controversy when assessing the associations between RASi treatment and breast cancer. A meta-analysis and systemic review found no association of ACEI/ARB use with disease-free or overall survival in breast cancer (28). Another recent systemic review from 2018 of 11 only observational studies, found evidence that beta-blockers but not ACEIs can reduce the risk of breast cancer recurrence (29).

Similarly, another recent review (18) reported that three out of 13 studies in breast cancer found worse outcomes and only two studies found beneficial effects. As demonstrated by Chae and colleagues among 168 ACEI users with a mean follow up of 55 months, a reduced risk of recurrence was found in patients with stage II/III breast cancer (30). Nonetheless, two long-term studies with large cohorts conducted in Taiwan (31) and more recently in the US (32) found a protective association with the use of ACEIs in patients with breast cancer. The Taiwan nationwide casecontrol analysis compared longitudinal use of ACEIs in 16,847 female patients with breast cancer and 50,541 matched individuals. The results showed the risk of developing breast cancer decreased as the ACEI dose increased (31). In a large cohort study of 90,078 postmenopausal ( $>55$ years) US women with hypertension, long-term treatment with ACEIs (2-12 years) had a protective association against breast cancer. This reduced occurrence was observed within 2-3 years of initiation of treatment with a rise after year 7-8 (32). Similarly, Li et al. also reported a reduction in breast cancer risk associated with long-term ( $>10$-year) use of ACEIs (33). The reduced breast cancer risk over time with ACEI use is related to the mechanism by which production of angiotensin II decreases soon after ACEI initiation, thus inhibiting angiogenesis and the growth of cancer. This is in agreement with the aforementioned studies, in which the protective association was observed relatively early.

\section{RASi Treatment Adjuvant to Chemotherapy}

Radin et al. reviewed some of the mounting clinical evidence demonstrating ACEIs have oncolytic activity in multiple types of cancer and discussed their ability to prevent cardiotoxicity of multiple chemotherapies (34). Their analysis demonstrated that the actions of ACEI converge on VEGF, reducing VEGF levels in tumors and preventing construction of blood vessels to masses, consequently leaving them nutrient-depleted, thus hindering tumor growth. Recent studies have assessed whether ACEI/ARB regimens combined with chemotherapeutic agents (e.g. with platinum-based chemotherapy or with anti-VEGFtargeted therapies) may improve patient outcomes in multiple types of cancer (e.g. lung, pancreatic, gastric, RCC). A metaanalysis of seven retrospective studies evaluated the effect of adjunctive RASi therapy combined with chemotherapeutic agents in a total of 2,436 patients with cancer (35). Their analysis showed that 378 patients who received ACEI/ARB plus chemotherapeutic agents had a significant reduction in 
overall mortality, in addition to a significant benefit in progression-free survival for the combination treatment. Subgroup analysis of 57/193 patients on platinum-based agents plus ACEI/ARB showed them to have a significant increase in survival outcome (35).

Concomitant RASi treatment with anti-VEGF-targeted therapies was also found to be associated with better survival. A recent review (18) on concomitant RASi treatment with platinum-based chemotherapy found in four out of five studies on patients with advanced non-small cell lung cancer led to a gain in overall survival of 3-5 months. Combination platinum-based agents plus ACEI/ARB treatment led to a gain of 5.7 months in overall survival in advanced gastric cancer in a study with 12.6 months follow up by Kim et al. (36). In metastatic CRC, Osumi et al. found up to 11 months gain in overall survival (37), while Engineer et al. found combination therapy reduced mortality and tumor progression in patients with CRC (38).

The aforementioned studies on metastatic RCC, by Keizman et al. (19) and McKay et al. (20) showed combination therapy led to a gain in overall survival of 7 to 26 months. In a study by Pinter et al. in patients with advanced HCC combination therapy led to a gain in overall survival of 5 months (39). Longer overall survival was also shown by Levin et al. with combination therapy in 318/1,186 patients with glioblastoma (40). Although neoadjuvant systemic therapy is often used in locally advanced and inflammatory breast cancer, ACEI use had no significant effect on survival as shown by Chae et al. among 1,449 patients with stage I-III breast cancer receiving neoadjuvant chemotherapy; only the 54 ARB users had reduced risk of recurrence, with a $82 \%$ relapse-free survival at 5 years (41). Similarly, in another study by Chae et al. no survival benefit was found with adjuvant chemotherapy in patients with acute myeloid leukemia (42). Nakai et al. also showed no survival benefit from RASi therapy with adjuvant chemotherapy in biliary tract cancer (43).

\section{Conclusion}

Commonly used medications for the treatment of hypertension such as ACEIs and ARBs that target and modulate the RAS system have been the focus of studies for more than two decades assessing whether they benefit the prognosis of patients with cancer. Evidence of such a positive association between ACEI/ARB treatment and reduced tumor growth has been demonstrated in experimental animal models. Additional evidence on a protective association between ACEI/ARBs treatment and incidence of specific cancer types has been accumulated from numerous human clinical studies (including large epidemiological studies, and long-term prospective and retrospective studies). Response to RASi therapy might be dependent on tumor type, characteristic or stage as retrospective studies show specific cancer types (namely RCC, non-small cell lung cancer, HCC, and PDAC) are more responsive to RASi treatment than others (e.g. breast cancer). Additionally, polymorphism in the ACE gene has been suggested to be associated with the susceptibility to develop certain cancer types. Moreover, with respect to cancer treatment, ACEI/ARB as co-adjuvants with chemotherapeutic agents (e.g. with platinum-based chemotherapy or with anti- VEGF-targeted therapies) have been suggested to prevent cardiotoxicity of multiple chemotherapies and, thus, improve patient outcomes (i.e. progression-free survival, and prolonged overall survival) in different types of cancer. Notably, treatment of the individual patient should be monitored regarding adverse events.

\section{Conflicts of Interest}

The Authors have no conflicts of interest to disclose.

\section{Authors' Contributions}

TR, IG Participated in the writing of the review and revised the final version for publication.

\section{References}

1 Wegman-Ostrosky T, Soto-Reyes E, Vidal-Millán $\mathrm{S}$ and Sanchez-Corona J: The renin-angiotensin system meets the hallmarks of cancer. J Renin-Angiotensin-Aldosterone Syst 16: 227-233, 2015. PMID: 23934336. DOI: 10.1177/14703203 13496858

2 Lucero HA, Kintsurashvili E, Marketou ME and Gavras H: Cell signaling, internalization, and nuclear localization of the angiotensin-converting enzyme in smooth muscle and endothelial cells. J Biol Chem 285: 5555-5568, 2010. PMID: 20022959. DOI: 10.1074/jbc.M109.074740

3 Yoshiji H, Kuriyama S, Noguchi R and Fukui H: Angiotensin-Iconverting enzyme inhibitors as potential anti-angiogenic agents for cancer therapy. Curr Cancer Drug Targets 4: 555-567, 2004. PMID: 15578913. DOI: 10.2174/1568009043332790

4 Lever AF, Hole DJ, Gillis CR, McCallum IR, McInnes GT, MacKinnon PL, Meredith PA, Murray LS, Reid JL and Robertson JWK: Do inhibitors of angiotensin-I-converting enzyme protect against risk of cancer? Lancet 352: 179-184, 1998. PMID: 9683206. DOI: 10.1016/S0140-6736(98)03228-0

5 Rosenthal $\mathrm{T}$ and Gavras $\mathrm{I}$ : Angiotensin inhibition and malignancies: A review. J Hum Hypertens 23: 623, 2009. PMID: 19339998. DOI: 10.1038/jhh.2009.21

6 Araújo WF, Naves MA, Ravanini JN, Schor N and Teixeira VPC: Renin-angiotensin system (RAS) blockade attenuates growth and metastatic potential of renal cell carcinoma in mice: Urol Oncol 33(9): 389.e1-7, 2015. PMID: 25595575. DOI: 10.1016/j.urolonc.2014.11.022

7 Yang K-K, Sui Y, Zhou H-R and Zhao H-L: Interaction of reninangiotensin system and adenosine monophosphate-activated protein kinase signaling pathway in renal carcinogenesis of uninephrectomized rats. Tumor Biol 39(5), 2017. PMID: 
28459365. DOI: $10.1177 / 1010428317699116$

8 Mansour MA, Al-Ismaeel H, Al-Rikabi AC and Al-Shabanah OA: Comparison of angiotensin-converting enzyme inhibitors and angiotensin II type 1 receptor blockade for the prevention of premalignant changes in the liver. Life Sci 89: 188-194, 2011. PMID: 21699905. DOI: 10.1016/j.lfs.2011.06.002

9 Koh SL, Ager EI, Costa PLN, Malcontenti-Wilson C, Muralidharan V and Christophi C: Blockade of the reninangiotensin system inhibits growth of colorectal cancer liver metastases in the regenerating liver. Clin Exp Metastasis 31: 395-405, 2014. PMID: 24442969. DOI: 10.1007/s10585-0149635-8

10 Friis S, Sørensen HT, Mellemkjær L, McLaughlin JK, Nielsen GL, Blot WJ and Olsen JH: Angiotensin-converting enzyme inhibitors and the risk of cancer: A population-based cohort study in Denmark. Cancer 92: 2462-2470, 2001. PMID: 11745304. DOI: 10.1002/1097-0142(20011101)92:93.0.

11 Mandilaras V, Bouganim N, Yin H, Asselah J and Azoulay L: The use of drugs acting on the renin-angiotensin system and the incidence of pancreatic cancer. Br J Cancer 116: 103, 2017. PMID: 27846200. DOI: 10.1038/bjc.2016.375

12 Makar GA, Holmes JH and Yang Y-X: Angiotensin-converting enzyme inhibitor therapy and colorectal cancer risk. JNCI J Natl Cancer Inst 106, 2014. PMID: 24431411. DOI: 10.1093/ jnci/djt374

13 Chiang Y-Y, Chen K-B, Tsai T-H and Tsai W-C: Lowered cancer risk with ACE Inhibitors/ARBs: A population-based cohort study. J Clin Hypertens 16: 27-33, 2014. PMID: 24304931. DOI: $10.1111 /$ jch.12228

14 Dai Y-N, Wang J-H, Zhu J-Z, Lin J-Q, Yu C-H and Li Y-M: Angiotensin-converting enzyme inhibitors/angiotensin receptor blockers therapy and colorectal cancer: A systematic review and meta-analysis. Cancer Causes Control 26: 1245-1255, 2015. PMID: 26081426. DOI: 10.1007/s10552-015-0617-1

15 Shen J, Huang Y-M, Wang M, Hong X-Z, Song X-N, Zou X, Pan Y-H, Ling W, Zhu M-H, Zhang X-X, Sui Y and Zhao HL: Renin-angiotensin system blockade for the risk of cancer and death. J Renin-Angiotensin-Aldosterone Syst 17(3), 2016. PMID: 27402638. DOI: 10.1177/1470320316656679

16 van der Knaap R, Siemes C, Coebergh J-WW, van Duijn CM, Hofman A and Stricker BHC: Renin-angiotensin system inhibitors, angiotensin I-converting enzyme gene insertion/ deletion polymorphism, and cancer: The Rotterdam Study. Cancer 112: 748-757, 2008. PMID: 18181094. DOI: 10.1002/ cncr.23215

17 Liu Y-T, Lin L-W, Chen C-Y, Wang C-P, Liu H-P, Houng J-Y, Chung F-M and Shieh T-Y: Polymorphism of angiotensin Iconverting enzyme gene is related to oral cancer and lymph node metastasis in male betel quid chewers. Oral Oncol 48: 1257-1262, 2012. PMID: 22776130. DOI: 10.1016/j.oraloncology. 2012.06.003

18 Pinter M and Jain RK: Targeting the renin-angiotensin system to improve cancer treatment: Implications for immunotherapy. Sci Transl Med 9: eaan5616, 2017. PMID: 28978752. DOI: 10.1126/scitranslmed.aan5616

19 Keizman D, Huang P, Eisenberger MA, Pili R, Kim JJ, Antonarakis ES, Hammers $\mathrm{H}$ and Carducci MA: Angiotensin system inhibitors and outcome of sunitinib treatment in patients with metastatic renal cell carcinoma: A retrospective examination. Eur J Cancer 47: 1955-1961, 2011. PMID: 21600760. DOI: 10.1016/j.ejca.2011.04.019
20 McKay RR, Rodriguez GE, Lin X, Kaymakcalan MD, Hamnvik O-PR, Sabbisetti VS, Bhatt RS, Simantov R and Choueiri TK: Angiotensin system inhibitors and survival outcomes in patients with metastatic renal cell carcinoma. Clin Cancer Res 21: 24712479, 2015. PMID: 25724518. DOI: 10.1158/1078-0432.CCR14-2332

21 Colt JS, Hofmann JN, Schwartz K, Chow W-H, Graubard BI, Davis F, Ruterbusch J, Berndt $\mathrm{S}$ and Purdue MP: Antihypertensive medication use and risk of renal cell carcinoma. Cancer Causes Control 28: 289-297, 2017. PMID: 28224412. DOI: 10.1007/s 10552-017-0857-3

22 Yoshiji H, Noguchi R, Ikenaka Y, Kaji K, Aihara Y, Yamazaki M, Yamao J, Toyohara M, Mitoro A, Sawai M and Yoshida M: Combination of branched-chain amino acids and angiotensinconverting enzyme inhibitor suppresses the cumulative recurrence of hepatocellular carcinoma: A randomized control trial. Oncol Rep 26: 1547-1553, 2011. PMID: 21874260. DOI: 10.3892/or.2011.1433

23 Walker AJ, West J, Grainge MJ and Card TR: Angiotensinconverting enzyme inhibitors and hepatocellular carcinoma incidence in the General Practice Research Database. Cancer Causes Control 22: 1743, 2011. PMID: 21909951. DOI: 10.1007/s 10552-011-9837-1

24 Blute Jr ML, Rushmer TJ, Shi F, Fuller BJ, Abel EJ, Jarrard DF and Downs TM: Renin-angiotensin inhibitors decrease recurrence after transurethral resection of bladder tumor in patients with nonmuscle invasive bladder cancer. J Urol 194: 1214-1219, 2015. PMID: 26173101. DOI: 10.1016/j.juro. 2015.05.104

25 Yoshida T, Kinoshita H, Fukui K, Matsuzaki T, Yoshida K, Mishima T, Yanishi M, Komai Y, Sugi M, Inoue T and Murota T: Prognostic impact of renin-angiotensin inhibitors in patients with bladder cancer undergoing radical cystectomy. Ann Surg Oncol 24: 823-831, 2017. PMID: 27730369. DOI: 10.1245/ s10434-016-5534-3

26 Alashkham A, Paterson C, Windsor P, Struthers A, Rauchhaus $\mathrm{P}$ and Nabi G: The incidence and risk of biochemical recurrence following radical radiotherapy for prostate cancer in men on angiotensin-converting enzyme inhibitors (ACEIs) or angiotensin receptor blockers (ARBs). Clin Genitourin Cancer 14: 398-405, 2016. PMID: 27053500. DOI: 10.1016/ j.clgc.2016.03.009

27 Liu H, Naxerova K, Pinter M, Incio J, Lee H, Shigeta K, Ho WW, Crain JA, Jacobson A, Michelakos T and Dias-Santos D: Use of angiotensin system inhibitors is associated with immune activation and longer survival in nonmetastatic pancreatic ductal adenocarcinoma. Clin Cancer Res, 2017. PMID: 28600474. DOI: 10.1158/1078-0432.CCR-17-0256

28 Raimondi S, Botteri E, Munzone E, Cipolla C, Rotmensz N, DeCensi A and Gandini S: Use of beta-blockers, angiotensinconverting enzyme inhibitors and angiotensin receptor blockers and breast cancer survival: Systematic review and meta-analysis. Int J Cancer 139: 212-219, 2016. DOI: 10.1002/ijc.30062

29 Zhao Y, Wang Q, Zhao X, Meng $\mathrm{H}$ and Yu J: Effect of antihypertensive drugs on breast cancer risk in female hypertensive patients: Evidence from observational studies. Clin Exp Hypertens 40: 22-27, 2018. PMID: 26916107. DOI: 10.1080/10641963.2017.1288736

30 Chae YK, Valsecchi ME, Kim J, Bianchi AL, Khemasuwan D, Desai A and Tester W: Reduced risk of breast cancer recurrence 
in patients using ACE inhibitors, ARBs, and/or statins. Cancer Invest 29: 585-593, 2011. PMID: 21936625. DOI: 10.3109/ 07357907.2011.616252

31 Lee H-H, Tsan Y-T, Ho W-C, Lin M-H, Lee C-H, Tseng C-D, Guo Y-L, Wang J-D, Chen P-C and Health Data Analysis in Taiwan (hDATa) Research Group HDA: Angiotensin-converting enzyme inhibitors enhance the effect of cyclooxygenase inhibitors on breast cancer: A nationwide case-control study. J Hypertens 30: 2432-2439, 2012. PMID: 23027181. DOI: 10.1097/HJH.0b013e328358d42f

32 Raebel MA, Zeng C, Cheetham TC, Smith DH, Feigelson HS, Carroll NM, Goddard K, Tavel HM, Boudreau DM, Shetterly S and Xu S: Risk of breast cancer with long-term use of calcium channel blockers or angiotensin-converting enzyme inhibitors among older women. Am J Epidemiol 185: 264-273, 2017. PMID: 28186527. DOI: 10.1093/aje/kww217

33 Li CI, Daling JR, Tang M-TC, Haugen KL, Porter PL and Malone KE: Use of antihypertensive medications and breast cancer risk among women aged 55 to 74 years. JAMA Intern Med 173: 1629-1637, 2013. PMID: 23921840. DOI:10.1001/ jamainternmed.2013.9071

34 Radin DP, Krebs A, Maqsudlu A and Patel P: Our ACE in the hole: Justifying the use of angiotensin-converting enzyme inhibitors as adjuvants to standard chemotherapy. Anticancer Res 38: 45-49, 2018. PMID: 29277755. DOI: 10.21873/anticanres.12190

$35 \mathrm{Li} \mathrm{XY,} \mathrm{Sun} \mathrm{JF} \mathrm{and} \mathrm{Hu} \mathrm{SQ:} \mathrm{The} \mathrm{renin-angiotensin} \mathrm{system}$ blockers as adjunctive therapy for cancer: A meta-analysis of survival outcome. Eur Rev Med Pharmacol Sci 21: 1375-1383, 2017. PMID: 28387887.

36 Kim ST, Park KH, Oh SC, Seo JH, Kim JS, Shin SW and Kim YH: How does inhibition of the renin-angiotensin system affect the prognosis of advanced gastric cancer patients receiving platinum-based chemotherapy? Oncology 83: 354-360, 2012. PMID: 23052034. DOI: 10.1159/000337979

37 Osumi H, Matsusaka S, Wakatsuki T, Suenaga M, Shinozaki E and Mizunuma N: Angiotensin II type-1 receptor blockers enhance the effects of bevacizumab-based chemotherapy in metastatic colorectal cancer patients. Mol Clin Oncol 3: 12951300, 2015. PMID: 26807236. DOI: 10.3892/mco.2015.630

38 Engineer DR, Burney BO, Hayes TG and Garcia JM: Exposure to ACEI/ARB and $\beta$-blockers is associated with improved survival and decreased tumor progression and hospitalizations in patients with advanced colon cancer. Transl Oncol 6: 539-545, 2013. PMID: 24151534. DOI: 10.1593/tlo.13346

39 Pinter M, Weinmann A, Wörns M-A, Hucke F, Bota S, Marquardt JU, Duda DG, Jain RK, Galle PR, Trauner M, Peck-Radosavljevic
$\mathrm{M}$ and Sieghart W: Use of inhibitors of the renin--angiotensin system is associated with longer survival in patients with hepatocellular carcinoma. United Eur Gastroenterol J 5: 987-996, 2017. PMID: 29163965. DOI: 10.1177/2050640617 695698.

40 Levin VA, Chan J, Datta M, Yee JL and Jain RK: Effect of angiotensin system inhibitors on survival in newly diagnosed glioma patients and recurrent glioblastoma patients receiving chemotherapy and/or bevacizumab. J Neurooncol 134: 325-330, 2017. PMID: 28631191. DOI: 10.1007/s11060-017-2528-3

41 Chae YK, Brown EN, Lei X, Melhem-Bertrandt A, Giordano SH, Litton JK, Hortobagyi GN, Gonzalez-Angulo AM and Chavez-MacGregor M: Use of ACE inhibitors and angiotensin receptor blockers and primary breast cancer outcomes. J Cancer 4: 549, 2013. PMID: 23983819. DOI: 10.7150/jca.6888

42 Chae YK, Dimou A, Pierce S, Kantarjian H and Andreeff M: The effect of calcium channel blockers on the outcome of acute myeloid leukemia. Leuk Lymphoma 55: 2822-2829, 2014. PMID: 24628293. DOI: 10.3109/10428194.2014.901513

43 Nakai Y, Isayama H, Sasaki T, Takahara N, Saito K, Takeda T, Umefune G, Saito T, Takagi K, Watanabe T, Hamada T, Uchino R, Mizuno S, Yamamoto K, Kogure H, Matsubara S, Yamamoto $\mathrm{N}$, Ijichi H, Tateishi K, Tada M and Koike K: No survival benefit from the inhibition of renin-angiotensin system in biliary tract cancer. Anticancer Res 36: 4965-4970, 2016. PMID: 27630357. DOI: 10.21873 /anticanres.11065
Received July 3, 2019

Revised July 17, 2019

Accepted July 22, 2019 